\title{
plicatura de la gran curvadura DEL estomago como CAUSA DE VOMITO EN EL LACTANTE
}

\author{
Por los Dres. ARMANDO DOBERTI, JORGE E. HOWARD. WERNER BUSTAMANTE \\ Y AUGUSTO WINTER \\ Hospital Luis Calvo Mackenna. Cátedra de Pediatria del Prof. A. Ariztía A.
}

Durante el curso de los dos últimos años, en el Hospital Luis Calvo Mackenna hemos procedido al estudio radiológico sistemático de todo lactante que presentare el síntoma vómito o sintoma regurgitación sin causa bien evidente. Con esta política hemos logrado pesquisar y estudiar una serie de cuadros, unos más, otros menos frecuentes, que antes se englobaban en el gran rubro de vómitos habituales del lactante, sin procederse, en la gran mayoría de los casos, a precisar más el diagnóstico. Es así como fuera de los cuadros bien conocidos como la hipertrofia del píloro, hemos podido diagnosticar casos de braquiesófago, de plicaturas del estó mago y de obstrucciones crónicas a nivel del duodeno, ya sea por mal rotaciones, bridas congénitas, pinza mesentérica y otras causas poco comunes. En esta oportunidad nos vamos a referir solamente a la plicatura del estómago, dejando para una próxima comunicación los cuadros restantes.

La plicatura del estómago, ya sea de la gran curvadura o de la totalidad del estómago es un síndrome que no es raro en el lactante, ya que en el transcurso de 2 años hemos logrado reunir 12 casos. Revisando la literatura nos encontramos con que, entre nosotros, Doberti ${ }^{1}$ presentó 2 casos a la sociedad Chilena de Radiología en la sesión del 23 de junio de 1950 bajo el título de "Vólvulos del estómago", y en la literatura mundial sólo hemos encontrado los artículos de Marie y col."2 y los de Ro- viralta" ${ }^{4}$, todios ellos refiriéndose solamente a la plicatura parcial de la gran curvadura, presentando los primeros 4 observaciones y 3 el segundo. Pensamos que el hecho que estos casos no se diagnostican con más frecuencia se deba a que se trata de un cuadro todavía mal conocido y a la falta del examen radiológico sistemático del lactante vomitador.

La sintomatología clínica se reduce a vómitos habituales, vómitos que en sí no tienen nada de característicos, pudiendo ser explosivos o fáciles y en algunas ocasiones ser simples regurgitaciones. LOS vómitos pueden aparecer a cualquiera hora del dia, con cualquiera alimentación, ya sea materna o artificial, en cualquiera posición que se coloque al niño y, además, no se observan ondas peristálticas. Los vómitos pueden comenzar en el período de recién nacido o más tarde y se entienđe que si se prolongan llevan a la distrofia, la cual es la acompañante obligada en los casos con algunos meses de evolución.

Es esencial para la visualización del cuadro radiológico iniciar el estudio gástrico con una pequeña cantidad de substancia opaca y movilizar al niño en varias posiciones, con el fin de impregnar la mucosa gástrica de la totalidad del estómago, para ver con nitidez la posición de sus curvaduras. $\mathrm{Si}$ en el examen de rutina se le da a ingerir una mamadera alimenticia mezclada con substancia de contraste y se inicia el examen radiológico sólo al final o des- 
pués de ingerida una parte de ella, tendremos solamente la imagen de un estómago grande transversal, ya que la cantidad de substancia de contraste no permite el análisis de los pliegues gástricos ni de las curvaduras, en forma correcta.

Iniciado el examen en la forma indicada, vemos en la plicatura parcial de la curvadura, que el estómago, al igual que en los casos de Marie y col. y de Roviralta, se presenta con un cardias en posición normal. El bario desciende hasta un primer segmento gástrico a izquierda, formado por el fornix con su ampolla de aire. La imagen se continúa a derecha por un segundo segmento, en el cual se pueden observar los pliegues gástricos, formando una ese itálica acostada, sucediéndole otro tanto a la curvadura mayor, es decir, que partiendo del formix a izquierda desciende, para luego ascender nuevamente en la región horizontal y hacerse superior en la región del cuerpo gástrico, descendiendo por el lado derecho en Ia región del antro. La curvadura menor, partiendo desde el cardias se dirige hacia abajo en un pequeño segmento vertical, luego después se hace horizontal, quedando bajo la curvadura mayor, que queda por encima y por delante, terminando luego después de un segundo segmento pequeño vertical, en la región del antro.

En la otra modalidad, la plicatura total de la gran curvadura, más extensa en cuanto al segmento de curvadura mayor evertida y que comparamos con un riñón acostado, la parte superior está formada por la curvadura mayor, que se dirige inmediatamente a derecha bajo el diafragma, formando una concavidad inferior con el estómago totalmente transversal. La curvadura menor en esta modalidad prácticamente no tiene el primer segmento vertical, ya que partiendo del cardias se hace inferior y horizontal, con una concavidad inferior de menor arco que la curvadura mayor $y$ que comparamos con la región del hilio renal. En esta variedad llama la atención que en las posiciones oblicuas solamente es posible ver el antro, el pílo- ro y el bulbo en posición oblicua anterior izquierdo y no en la posición habitual de oblicua anterior derecha.

La sintomatología radiológica de la plicatura se entiende mejor al estudiar su mecanismo de producción. En el primer caso, en la plicatura parcial de la gran curvadura igual que en los casos descritos por Roviralta y Marie la posición horizontal de la gran curvadura, el antro y el píloro se elevan, describiendo un ángulo de $180^{\circ}$ hacia adelante y arriba, con lo que estas porciones se colocan ahora por delante de la pequeña curvadura y sus caras posteriores pasan a ser anteriores y vice-versa. En la segunda variedad o plicatura total de la gran curvadura, el gran fondo va a quedar como límite superior convexo y la pequeña curvadura como límite inferior cóncavo de la imagen gástrica.

En cuanto al funcionamiento gástrico llama la atención que el peristaltismo no se efectúa en el estómago en forma normal, ya que sólo se observan contracciones segmentarias preferentemente del antro. Además, se observa una disfunción pilórica, observándose un pasaje casi continuo al comienzo del examen, para después cerrarse por un período más largo.

Saber por qué se producen estas plicaturas es ya más difícil y existen varias hipótesis que tratan de explicar el mecanismo de producción. Así podríamos suponer que se debiera a una mayor elongación de ciertos mesos, especialmente un mesocolon, que permitiera mayor movilidad al estómago, o bien, al hecho que en estos lactantes se acumulara gran cantidad de aire en el colon transverso, con lo cual este órgano tendería a colocarse en la parte más alta de la cavidad abdominal, arrastrando en su trayectoria al estómago. Si lo que ascendiera fuera el ángulo hepático, se produciría solamente la plicatura de la gran curvadura, mientras que en aquellos casos en que el meteorismo fuera tan intenso que todo el colon transverso ascendiera, se produciría la plicatura total del estómago. Como una última hipótesis quedaría suponer que estas alteraciones de 


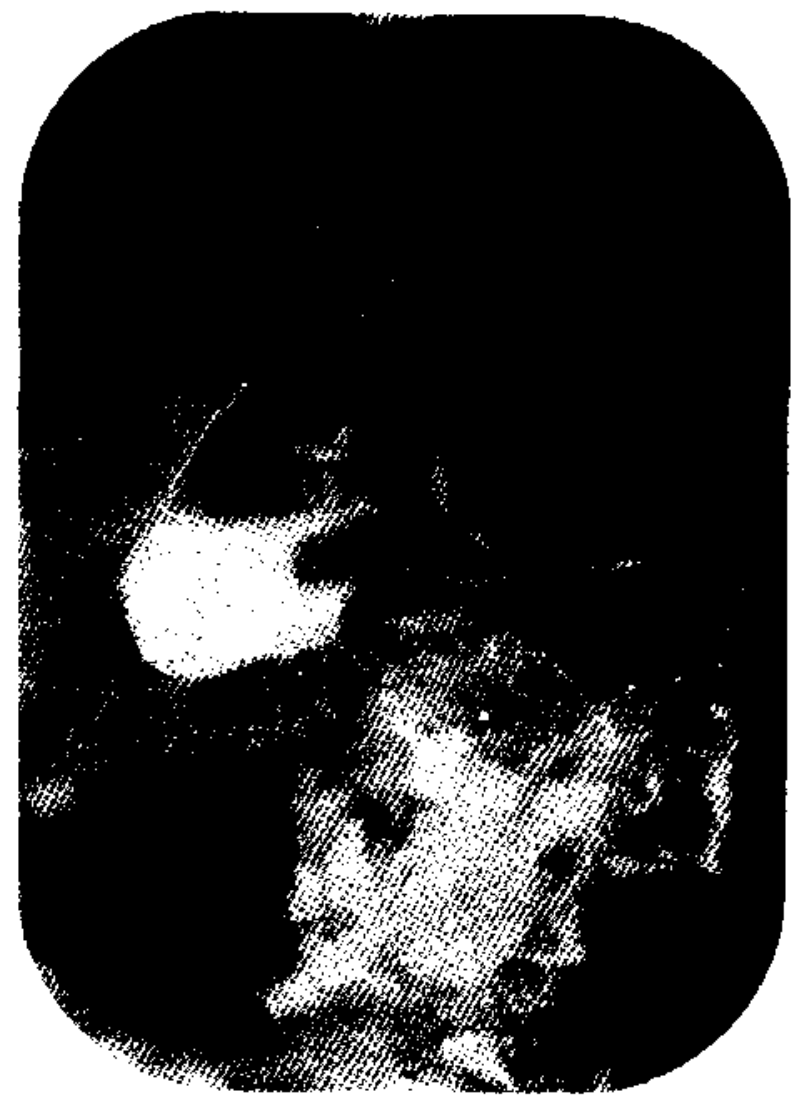

Plicatura totel.

J. C

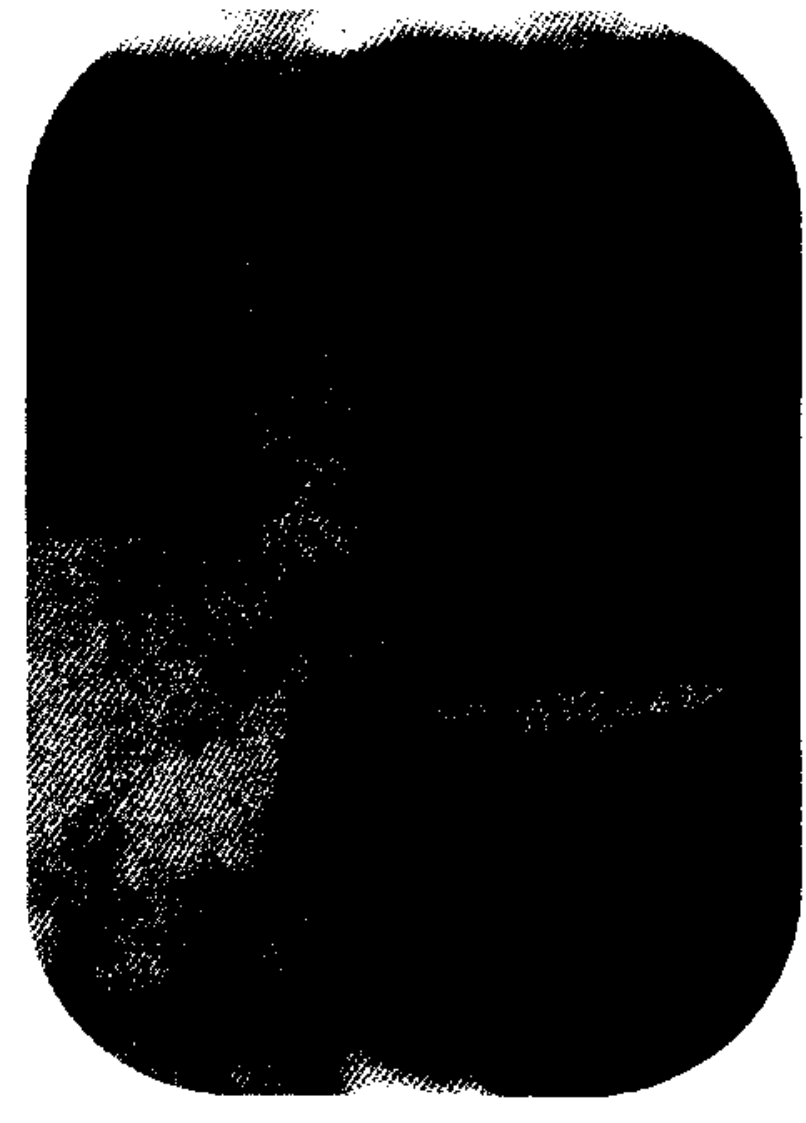

Plicatura parcial.

E. C. 


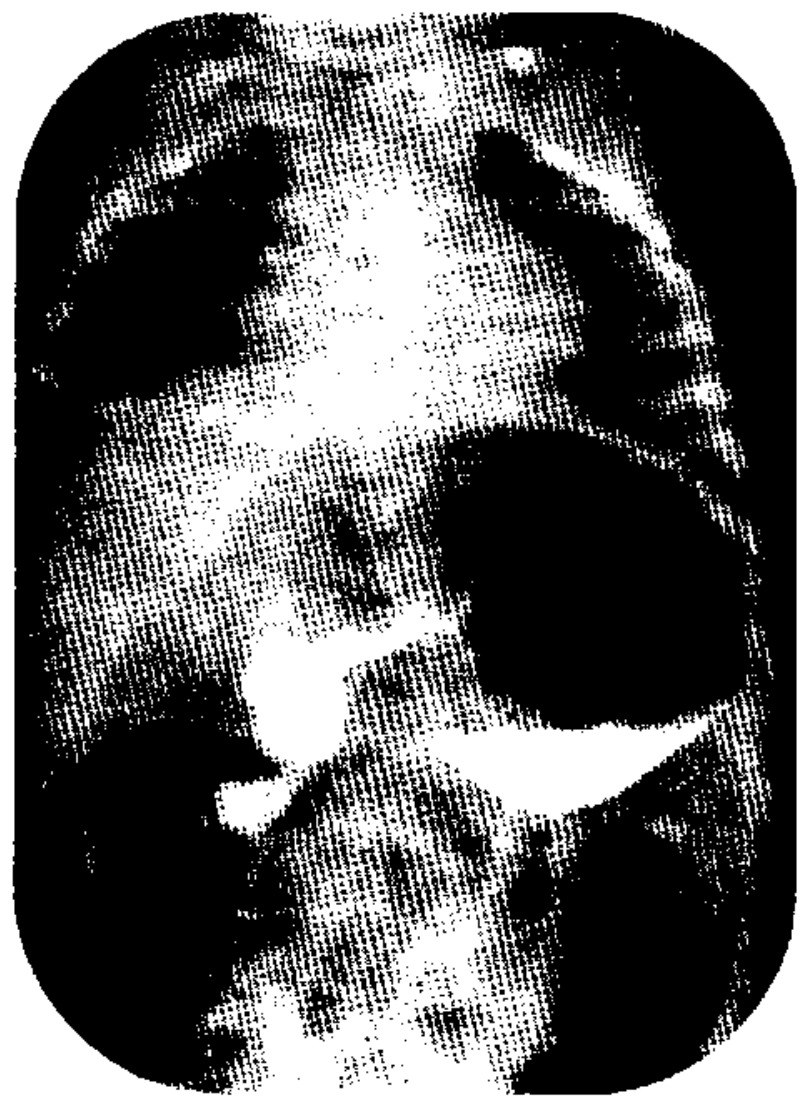

Plicatura parcial.

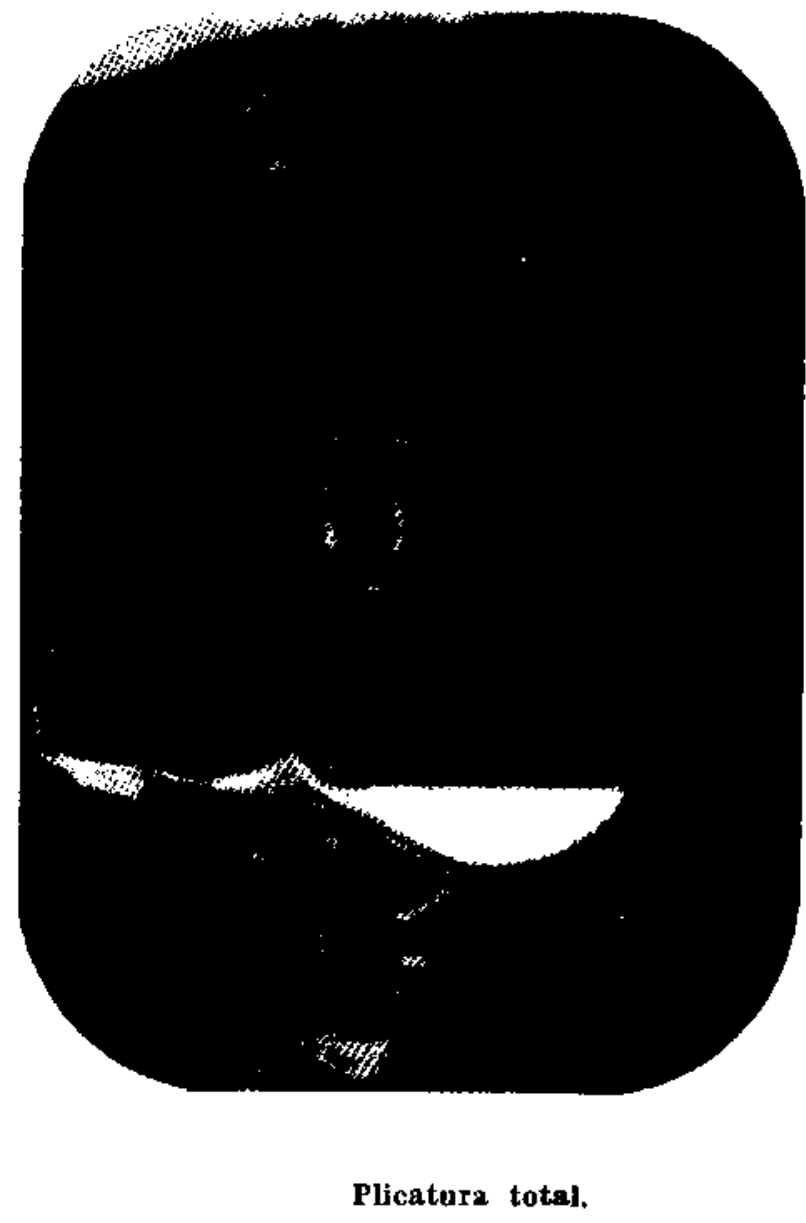

(ver posición del cardfas).

M. R.

R. A. 
la morfología gástrica fueran debidas a parálisis diafragmática.

Material de estudio: Nuestro material de estudio está constituído por 9 lactantes del sexo masculino y 3 del sexo femenino, cuyas edades fluctuaban al ingreso al hospital entre el mes 22 días y los 5 meses. El motivo de ingreso fué el síntoma vómito repeticio en 10 lactantes, en uno una tráqueobronquitis y en
Casos clínicos: Solamente vamos a dar el resumen de 3 observaciones clínicas, ya que todas ellas son sensiblemente iguales.

CASO 1. - Lactante del sexo femenino, de 3 meses de edad, con un peso de $3,460 \mathrm{~g}$, que presenta desde el mes de edad vómitos, en algunas ocasiones explosivas, en otras regurgitaclones, relaclonado con la ingestión de leches deshidratadas. no presentándose cuando ingería leche materna o mercenaria. Nacida

Cuadro $\mathbf{N}^{\circ} 1$.

\begin{tabular}{|c|c|c|c|c|c|c|}
\hline Obs. & sexo & $\begin{array}{l}\text { Comienzo } \\
\text { de los } \\
\text { vómitos }\end{array}$ & $\begin{array}{l}\text { alimen- } \\
\text { tación } \\
\text { materna }\end{array}$ & $\begin{array}{c}\text { Edad } \\
\text { al } \\
\text { ingreso }\end{array}$ & $\begin{array}{c}\text { Peso } \\
\text { al } \\
\text { ingreso }\end{array}$ & $\begin{array}{l}\text { Diagnóstico } \\
\text { al ingreso }\end{array}$ \\
\hline M.H.F. & $m$ & 2 dias. & 4 meses & 5 meses & 5450 & vomitador \\
\hline S.C. & $f$ & 30 dias & 1 mes & 3 meses & 3460 & vomitador \\
\hline E. A.F & m & 90 dias & 15 dias & 3 meses & 3220 & vomitador \\
\hline R.C.R. & $\mathrm{m}$ & ldia & 8 días & $1 \%$ mes & 3200 & vomitador \\
\hline R.A.S. & $m$ & 1 dia & 15 dias & 3 meses & 2500 & vomitador \\
\hline J.C.G. & $\mathrm{m}$ & 15 dias & 2 meses & 2 meses & 2920 & piloroespas \\
\hline J.P.J. & $\mathrm{m}$ & 30 dias & 30 días & $1 / / 2$ mes & 2550 & vomitador \\
\hline C. T. 0 & $\mathrm{~m}$ & 60 dias & 60 dias & 4 meses & 3320 & vomitador \\
\hline M.B.F. & $f$ & $?$ & 28 dias & 5 meses & 2650 & vomitador \\
\hline R. $M$. & $m$ & 25 dias & $?$ & 5 meses & 4500 & piloroespas \\
\hline M. L. & $f$ & 55 días & 35 dias & 2 meses & 5200 & vomitador \\
\hline M. R. & $m$ & 60 días & Odia & $21 / 2$ meses & 3420 & $\begin{array}{l}\text { premāturo } \\
\text { traqueobron } \\
\text { quitis }\end{array}$ \\
\hline
\end{tabular}

otro solamente una distrofia, pero este último presentó vómitos repetidos durante su hospitalización, lo que motivó el examen radiológico. En 3 se iniciaron los vómitos al nacimiento, en otros 4 antes del mes de edad. El diagnóstico de ingreso en los 10 lactantes que traían une historia de vómitos, fué el de vomitador habitual a píloroespasmo. Diez de los 12 lactantes eran distróficos. Al examen físico de ingreso, fuera de los signos propios de una distrofia, sólo se observó un meteorismo más o menos intenso en algunos casos (ver cuadro No 1 ). de parto eutósico de término. Alimentada al pecho materno un mes y luego Eleđón, Nestalba, Pelargón y leche humana mercenaria. AI ingreso se hacen los diagnósticos de: vomitador, distrofia y cardlopatia congénita aclanótica. Al examen fisico se encuentra un lactante en malas condiciones generales y nutritivas, pálido, sin cianosis, con un soplo hoIosistólico mesocardíaco $\sin$ irradiaciones, $y$, además, un intenso meteorismo. El examen radiológico revela una plicatura parcial de la gran curvadura y una rotación incompleta del cjego. Evolución: a pesar de alimentarla con leche humana, presenta frecuentes $\nabla \hat{o}^{-}$ mitos y regurgitactones y meteortsmo., Después de 3 meses y por seguir en iguales, condiciones, $\sin$ aumentar de peso, es sometida 
a una intervención quirúrgica, en la que se encuentra un mesenterio común laxo $y$ un ciego móvll que se fjja. Recuperada de su post-operatorlo, se somete a la cura postural recomendada por Roviralta, observándose una franca disminución de sus vómitos y un aumento apreciable de peso, ya que en 40 dias aumenta $1,200 \mathrm{~g}$. Un nuevo estudio radiológico de control hecho 70 dias después de la operación evidencia la persistencia de la plicatura.

CASO 2. - Lactante del sexo masculino, que Ingresa a la edad de 3 meses con un peso de $3,220 \mathrm{~g}$, con el diagnóstico de distrofia. Nacido de parto normal con un peso de 2,890 g, alimentado al pecho durante 15 dias, luego con Eledón. Al examen físico se aprecia un lactante abtrófíco. Evolución: por presentar vómitos y regurgltaciones se hace un estudio radiológlco, que demuestra una plleatura to. tal, que desaparece momentáneamente mientras el estómago está repleto de bario, para volver a producirse al estar éste vacío. A medida que se repara su distrofla se observa una tendencia a la disminución de las regurgitaciones y vómltos. Es dado de alta a los 8 meses con un peso de $\mathbf{5 , 5 0 0} \mathrm{g}$. En un control hecho a los 17 meses de edad persiste la plicatura total de la gran curvadura, ahora asoclada a una calasia del esófago.

CASO 3. - Lactante del sexo masculino, que ingresa a la edad de 3 meses con un peso de $2,500 \mathrm{~g}$, por presentar vómitos persistentes y rechazo de las mamaderas desde el periodo de recién nacido, síntomas que lo han lleyado a la distrofia. Nacldo de parto normal con un peso de $2,900 \mathrm{~g}$, alimentado ai pecho 8 dias, Al examen de ingreso se constata una plicatura total de la gran curvadura. Evolución: al ir progresando de peso disminuyen algo los vómitos y regurgitaciones, llamando la atención la gran lentitud de la reparación de la distrofia. En controles a los 2 y 4 meses despues del crimer examen radiológico persiste siempre la plicatura total. Es dedo de alta 5 meses después del ingreso, habiendo aumentado 2 kilos.

Comentario: El hecho que hayamos podido reunir 12 casos entre material de lactante de un solo hospital en plazo de 2 años, nos está indicando, como decíamos más atrás, que seguramente estos casos son más frecuentes de lo que a primera vista parece. Nos ha lamado la atención la predominancia de lactantes del sexo masculino, 9 de 12 casos. Solamente anotamos este hecho, ya que 12 casos no nos permiten deducir conelusio- nes valederas, pero parangonamos este hecho con lo que ocurre en la hipertrofia del píloro, en que según varios autores", el $75 \%$ de los lactantes que presentan este cuadro son varoncitos.

La sintomatología de estos casos se ha reducido principalmente a vómitos $\mathrm{y}$ regurgitaciones con las características ya anotadas de no tener ninguna particularidad especial. Pueden comenzar desde el nacimiento y si persisten un tiempo largo llevan a la distrofia, como ocurrió en la mayoría de estos enfermos. El estudio radiológico con ingestión de bario es esencial para establecer el diagnóstico y, además, nos permite el diagnóstico diferencial que si hay un pasaje esofágico normal se descarta el braquiesófago y si no hay alteraciones en el vaciamiento se descarta un síndrome pilórico o una estenosis duodenal, cuadros que producen también vómitos habituales en el lactante. Debemos consignar un hecho radiológico interesante ya señalado por Roviraita, que pudimos comprobar en 1 caso $\mathrm{y}$ es que el volvulus puede desaparecer durante el examen baritado, debido al peso del bario ingerido. En la patogenia de estas plicaturas podemos descartar la posibilídad que fueran debidas a parálisis diafragmáticas, ya que en todos los casos los diafragmas tenian movilidad normal. Nos parece más probable que en Ia producción del cuadro intervengan dos factores: la aereocolia, la cual sería la causante del ascenso del co. lon transverso, el cual en su trayectoria arrastraría al estómago, produciéndose así el volvulus parcial o el total y una cierta laxitud congénita de los ligamentos y mesos que mantienen al estomago en su posición normal. Si solamente interviniera el factor aereocolia, esta deformación del estómago debería presentarse más frecuentemente, sobre todo en los casos de mega y dolicocolon, lo cual no lo hemos observado en los varios casos de estas malformaciones intestinales, que hemos estudiado en el lactante. Además, la prueba terapéutica de colocar al lactante en posición de decúbito prono y en ligero Trendelenburg, posición preconizada por Roviralta como de rigor en tra- 
tamiento de la plicatura de la gran cur. vadura, por cuanto obligaría al colon transverso distendido a volver a la región media abdominal, solamente hemos tenido un éxito brillante en 2 de nuestros casos, lo que nos permite concluir que en ciertos casos, sin negar la importancia de la aereocolia, existen otros factores que juegan un rol primordial. Entre éstos estarían ciertas malformaciones anatómicas de los mesos, que facilitarían la producción del vómito. En nuestra casuística tenemos 3 casos que, además presentabar anomalías del tubo digesti- clínica, por el hecho que hemos visto períodos de exacerbación de los vómitos, persistiendo siempre la anomalía radiológica.

No sabernos cuanto tiempo dura este cuadro. El cuadro 2 nos indica el tiempo de control posterior de nuestros enfermos.

Solamente un caso falleció y a la autopsia se encontró un estómago en posición normal, que presentaba una escotadura en la región del antro y una muy discreta hipertrofia de las fibras musculares a nivel del píloro.

Cuadro No 2.

\begin{tabular}{|c|c|c|}
\hline Obs. & $\begin{array}{c}\text { Controlados } \\
\text { a los: }\end{array}$ & Comentarios: \\
\hline M. H.F. & no fué controlado & . \\
\hline S.C. & -4 meses & Sin vomitos. vólvulus \\
\hline E.A.F. & 14 meses & Vólvulus más calasia \\
\hline R. C.R. & 7 meses & normal \\
\hline R. A.S. & 5 meses & vólvulus \\
\hline J. C. G. & 2 meses. & vólvulus \\
\hline J.P. J. & 4 meses & Sin vómitos. Vólvulus \\
\hline C. T. Q. & fallece & \\
\hline M. B. F. & 3 meses & Vólvulus \\
\hline A. $M$. & - & $\cdots$ \\
\hline M. L. & $=$ & \\
\hline$\overline{M . R}$. & 2 meses & normal \\
\hline
\end{tabular}

vo: dos casos de malformación del intestino y 1 caso de una calasia del esófago del tipo de las descritas por Neuhauser y Beremberg ${ }^{\natural}$.

El que se presente uno $u$ otro tipo de plicatura depende de la mayor o menor extensión y elevación cólica, pudiendo un mismo enfermo presentar en exámenes distintos las dos variedades de plicatura. No podemos negar que, además de estos factores anatómicos deba existir un componente funcional importante en la producción de la sintomatología
En lo referente al tratamiento podemos concluir que debe ser combinado dietético y postural. El dietético deberá hacerse a base de una alimentación espesada, en algunos casos comidas pequeñas y frecuentes y el postural se hará colocando al lactante en la posición recomendada por Roviralta. Cuando pensamos que se agrega un factor espasmódico ręcurriremos a los sedantes y los antiespasmódicos. A pesar de las medidas enunciadas hay casos en que los vómitos y la sintomatología radiológica 
persisten, debiendo entonces, sobre todo si existe la malformación intestinal concomitante, pensar en la posibilidad de un tratamiento quirúrgico.

\section{Resumen.}

Se presentan 12 casos de vómitos habituales del lactante por plicatura parcial y total de la gran curvadura del estómago. Este sindrome está constituído por vómitos habituales $\mathrm{y}$ por una imagen radiológica anormal del estómago. Existen dos tipos de plicatura, parcial y total, modalidad esta úllima de la cual no encontramos referencias en la literatura. El tratamiento es mixto postural, dietético $\mathrm{y}$ en algunos casos aún quirúrgico.

\section{Summary.}

The authors study 12 infants with habitual vomiting due to a partial or total plicature of the greater curvature of the stomach. This is a clinal-radiological syndrome with habitual vomiting and an abnormal gastric $\mathrm{X}$ ray picture. There are two types of plicatures a partial variety and a total one. The authors have not found any bibliographical references to this second type of plicature. Treatment is postural, dietetic and in some cases surgical.

\section{Bibliografía.}

1.-DOBERTI, A. - volvulus del estómago. Sesićn del 23 de junio de 1950 de la Socledad Chllena de Radiologia.

2.-MARIE, J.; MIGNON, M.; SEE, GEORGES y MARTIN DU PAN, R. - Sur una varieté de vomissements habtuels du nourrisson par plicature de la grande courbure de l'stomac. Archives Francalses V: 274, 1948.

3.-ROVIRALTA, EMILIO. - La plicature de la grande courbure de l'stomac. Archives Francaise de Pediatrie VII: 72.5, 1950.

4.-ROVIRALTA, EMILIO. - Sur quelques causes des vomissements dits "habituels" du nourrisson. Helvética Pediatrica Acta IV: 445,1949 .

5.-WALUGREN, A. - Tuberculosis and other problems of pediatrics. Page 88. Willams and Wilkins Ed. 1951.

6.-BEREMBERG, W. and NEUHAUSER, E. -Cardioesophageal relaxation (chalasia) as cause of vomiting in infants. Pediatrles 5: $414,1950$. 